\title{
Human Immunodeficiency Virus Infection (HIV)/ Acquired Immunodeficiency Syndrome (AIDS): the frontiers and global challenges
}

HIV/AIDS continues to be a major health threat. An estimated 2.7 million new infections and an estimated 2 million deaths from HIV/AIDS-related illnesses were demonstrated in 2007. The World Health Organization (WHO) estimates that 8-10 million new cases of tuberculosis (TB) globally occur each year and accounts for $25 \%$ of AIDS deaths. Although AIDS is the same disease as HIV disease in all part of the world, this microorganism is mostly in many tropical countries. In tropical countries, TB and bacterial pneumonia represent the major pulmonary infections among the patients with HIVinfection/AIDS. Although the spectrum of HIV disease/AIDS is quite broad, the majority of the pulmonary infections in HIV-1 infected patients are similar to those observed in non-HIV infected persons. The geographical differences are primarily due to varying frequencies rather than the kinds of infections. Of all the pulmonary infections encountered in the tropics obviously Mycobacterium tuberculosis is one of the most significant pathogenic microorganisms. Great effort and resources have been invested in the improvement of diagnostic and treatment approaches for TB, and in identification of the close association between the two diseases. The developments have simultaneously benefited many living with HIV. Not all co-infections, nevertheless, have received sufficient attention. Donated funds would require paying for AIDS treatment, and funds remain insufficient for prevention strategies. Making therapy a priority could have the result of reducing fund, thus, increasing the numbers of cases which need therapy. When treatment and prevention ideals meet reality, prevention must be the highest priority. The United States Centers for Disease Control and Prevention (CDC) has recommended HIV testing of all pregnant women as part of the basic package of prenatal services for nearly two decades. Unfortunately, in the past, the focus of global mother-to-child HIV prevention efforts was on saving babies. What this policy meant in practice was that an HIV-positive pregnant woman who had a high CD4 count was given to save her baby only. Changes to this policy are underway in many countries. Clinical trials of microbicides to prevent HIV transmission in women have proved to be disappointing, nevertheless, a study in a simian
Volume I Issue 2 - 2014

\author{
Attapon Cheepsattayakorn,' Ruangrong \\ Cheepsattayakorn ${ }^{2}$ \\ 'IOth Zonal Tuberculosis and Chest Disease Center, \\ Department of Disease Control, Thailand \\ ${ }^{2}$ Department of Pathology, Faculty of Medicine, Chiang Mai \\ University, Thailand
}

Correspondence: Attapon Cheepsattayakorn, I0th Zonal Tuberculosis and Chest Disease Center, I 43 Sridornchai Road Changklan Muang Chiang Mai 50I00 Thailand,Tel 66-53-276364, Fax 66-53-273590, Email attaponche@yahoo.com

Received: June 09, 2014 | Published: June 10, 2014

immunodeficiency virus (SIV) rhesus macaque model pointed that the virus can be suppressed with a compound that prevents the recruitment of the very immune cells that the virus targets for infection. Although the study of a new vaccine in monkeys pointes that the T-cell-based strategies remain potentially viable, a major re-evaluation of the AIDS vaccine has been promptly performed. HIV/AIDS should be though about early in the development of drugs for important co-infections. Drug trials and designs must include patients with HIV/AIDS, where possible to avoid lags in treatment availability for common coinfections.

\section{Acknowledgment}

None.

\section{Conflict of interest}

None. 